Acta Crystallographica Section C

Crystal Structure

Communications

ISSN 0108-2701

\section{Intermolecular hydrogen bonding of the two independent molecules of $\mathrm{N}$-3,5-dinitrobenzoyl-L-leucine}

\author{
John F. Gallagher,* Peter T. M. Kenny and Michael \\ O’Donohoe
}

School of Chemical Sciences, Dublin City University, Dublin 9, Ireland Correspondence e-mail: gallagherjfg@dcu.ie

Received 17 February 2000

Accepted 2 March 2000

\section{Data validation number: IUC0000061}

The title compound, $\mathrm{C}_{13} \mathrm{H}_{15} \mathrm{~N}_{3} \mathrm{O}_{7}$, crystallizes as two independent molecules which differ in their conformation. Intermolecular hydrogen bonding between the amide and carboxylic acid groups as $\mathrm{N}-\mathrm{H} \cdots \mathrm{O}=\mathrm{C}$ interactions results in the formation of one-dimensional chains with $\mathrm{N} \cdots \mathrm{O}$ distances of 2.967 (6) and 3.019 (6) $\AA$. Neighbouring chains are linked by $\mathrm{C}=\mathrm{O} \cdots \mathrm{H}-\mathrm{O}$ interactions to form a twodimensional network, with $\mathrm{O} \cdots \mathrm{O}$ distances of 2.675 (6) and 2.778 (6) ^.

\section{Comment}

The study of biologically important molecules continues to be of primary importance in medicinal chemistry. Processes such as blood coagulation, hormone processing, viral replication and cancer-cell invasion are critically dependent on protease enzymes which are attractive target molecules in the design of specific and selective drugs. Important protease inhibitors are usually based on modified amino acids incorporating structural features which determine normal enzyme-substrate recognition processes. Structure-based drug design strategies to identify interactions between a potential inhibitor and target receptor require accurate inhibitor structures. We are currently studying structure-activity relationships and molecular-recognition processes in biologically important molecules such as the title compound $N$-3,5-dinitrobenzoyl-Lleucine, (I), for applications in drug design (Gallagher \& Murphy, 1999; Gallagher et al., 1999, 2000).

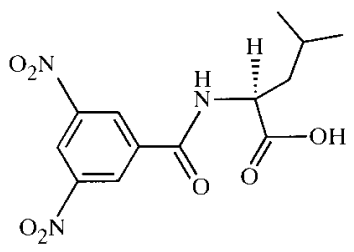

(I)
In (I), two independent molecules are present which differ in conformation in space group $P 1$ (No. 1). The bond lengths are largely in accord with anticipated values (Orpen et al., 1994). The molecules differ in conformation about the N1-C2 bond, with $\mathrm{N} 1-\mathrm{C} 2-\mathrm{C} 4$ angles of 115.9 (5) and $110.1(5)^{\circ}$, and $\mathrm{C} 1-\mathrm{N} 1-\mathrm{C} 2-\mathrm{C} 3$ torsion angles of $-56.1(7)$ and $81.5(7)^{\circ}$ in $A$ and $B$, respectively. The aromatic $\mathrm{C} 1-\mathrm{C} 11-\mathrm{C} 12$ and $\mathrm{C} 1-$ C11-C16 angles are $123.1(6) / 116.7(6)^{\circ}$ in molecule $A$ and $125.6(5) / 116.6(5)^{\circ}$ in molecule $B$. This results from the intramolecular contacts $\mathrm{H} 16 A \cdots \mathrm{O} 5 A \quad 2.45 \AA$ and $\mathrm{H} 1 A \cdots \mathrm{H} 12 A 2.08 \AA$ in $A$, and $\mathrm{H} 16 B \cdots \mathrm{O} 5 B 2.42 \AA$ and $\mathrm{H} 1 B \cdots \mathrm{H} 12 B 2.14 \AA$ in $B(\mathrm{O} 5 B \cdots \mathrm{H} 2 B 2.48 \AA)$. The aromatic ring planes are oriented at angles of $19.1(4)(A)$ and $12.8(6)^{\circ}$ $(B)$ to their respective amide groups $(\mathrm{O} 5 / \mathrm{C} 1 / \mathrm{N} 1 / \mathrm{C} 2)$ and the nitro groups are almost coplanar $\left(<10^{\circ}\right.$ from the aromatic ring planes), with O4A deviating by 0.265 (11) $\AA$ from the $\mathrm{C}_{6}$ ring plane. The carboxylate $(\mathrm{C} 2 / \mathrm{C} 3 / \mathrm{O} 6 / \mathrm{O} 7)$ groups are almost normal to the $(\mathrm{C} 11 / \mathrm{C} 1 / \mathrm{O} 5 / \mathrm{N} 1 / \mathrm{C} 2)$ planes, with angles of 85.9 (2) and 79.1 (2) $)^{\circ}$ in molecules $A$ and $B$, respectively.

The molecules are aligned in one-dimensional chains as $[A \ldots]_{n} \quad$ and $\quad[B \ldots]_{n}$ with $(\text { amide })_{[A / B]} \mathrm{N}-\mathrm{H} \cdots \mathrm{O}=$ $\mathrm{C}_{[A / B]}$ (acid) hydrogen bonds and partial overlap due to $\pi-\pi$ stacking of the 3,5-dinitrobenzoyl groups. The $\mathrm{N}$. . O distances are 2.967 (6) $\AA$ along $[A \ldots]_{n}$ and 3.019 (6) $\AA$ along $[B \ldots]_{n}$. Interactions between the neighbouring $A$ and $B$ chains arise as carboxylate (for $A \rightarrow B$ ) and 2.778 (6) $\AA$ (for $B \rightarrow A$ ) (where $\rightarrow$ indicates the direction of the hydrogen bonding). This association results in the formation of two 20-membered hydrogen-bonded ring systems each consisting of two $A$ and two $B$ molecules and differing by the direction of the hydrogen bonding, $[B \ldots]_{n} \rightarrow[A \ldots]_{n}$ and $[A \ldots]_{n} \rightarrow[B \ldots]_{n}$ (where $\rightarrow$ indicates the carboxylic acid to amide $\mathrm{O}=\mathrm{C}$ hydrogen bonds). Both rings have graph-set $R_{4}^{4}(20)$ which repeats as a two-dimensional network in the lattice (rings $J$ and $K$ in the deposited figure). Examination of the structure with PLATON (Spek, 1998) showed that there were no solventaccessible voids.

The structure of L-leucine has been reported previously (Harding \& Howieson, 1976; Collect et al., 1986; Görbitz \& Dalhus, 1996) and contains two crystallographically independent zwitterions having similar conformations in the asymmetric unit, unlike (I) above, where the two molecules differ significantly in conformation.

\section{Experimental}

$N$-3,5-Dinitrobenzoyl-L-leucine was synthesized by the reaction of 3,5-dinitrobenzoyl chloride with the parent L-leucine. Recrystallization from ethanol/water afforded colourless plate-like crystals suitable for X-ray analysis [m.p. 451-453 K (uncorrected); literature $187^{\circ}$ (Vogel, 1989)]. IR $v_{\max }(\mathrm{KBr}): 3400,1725,1650,1550,1350 \mathrm{~cm}^{-1}$. ${ }^{1} \mathrm{H}$ NMR data (400 MHz, $\delta$, DMSO): $0.87-0.94[6 \mathrm{H}, d, J=6.4 \mathrm{~Hz}$, $\left.\mathrm{C}\left(\mathrm{CH}_{3}\right)_{2}\right], 1.59-1.82\left(3 \mathrm{H}, m, \mathrm{CH}_{2} \mathrm{CH}\right), 4.47-4.53\left(1 \mathrm{H}, m, \mathrm{NCHCO}_{2}\right)$, $8.38(1 \mathrm{H}, d, J=7.9 \mathrm{~Hz}, \mathrm{NH}), 8.96(1 \mathrm{H}, t, J=2 \mathrm{~Hz}$, ArH-para $), 9.10$ $(2 \mathrm{H}, d, J=2 \mathrm{~Hz}$, ArH-ortho). 


\section{Crystal data}

$$
\begin{aligned}
& \mathrm{C}_{13} \mathrm{H}_{15} \mathrm{~N}_{3} \mathrm{O}_{7} \\
& M_{r}=325.28 \\
& \text { Triclinic, } P 1 \\
& a=5.8046(3) \AA \\
& b=10.6400(17) \AA \\
& c=12.9556(14) \AA \\
& \alpha=109.428(11)^{\circ} \\
& \beta=102.416(7)^{\circ} \\
& \gamma=90.250(8)^{\circ} \\
& V=734.44(15) \AA^{3}
\end{aligned}
$$

\section{Data collection}

Enraf-Nonius CAD-4 diffractometer

$\omega-2 \theta$ scans

2621 measured reflections

2621 independent reflections

1326 reflections with $I>2 \sigma(I)$

$\theta_{\max }=25.0^{\circ}$

\section{Refinement}

Refinement on $F^{2}$

$R\left[F^{2}>2 \sigma\left(F^{2}\right)\right]=0.045$

$w R\left(F^{2}\right)=0.079$

$S=0.937$

2621 reflections

418 parameters

$\mathrm{H}$-atom parameters constrained

$w=1 /\left[\sigma^{2}\left(F_{o}^{2}\right)+(0.0242 P)^{2}\right]$

where $P=\left(F_{o}^{2}+2 F_{c}^{2}\right) / 3$

Table 1

Selected geometric parameters $\left(\AA{ }^{\circ}\right)$.

\begin{tabular}{llll}
\hline $\mathrm{O} 1 A-\mathrm{N} 13 A$ & $1.226(6)$ & $\mathrm{O} 1 B-\mathrm{N} 13 B$ & $1.220(6)$ \\
$\mathrm{O} 2 A-\mathrm{N} 13 A$ & $1.232(6)$ & $\mathrm{O} 2 B-\mathrm{N} 13 B$ & $1.230(6)$ \\
$\mathrm{O} 3 A-\mathrm{N} 15 A$ & $1.237(7)$ & $\mathrm{O} 3 B-\mathrm{N} 15 B$ & $1.218(6)$ \\
$\mathrm{O} 4 A-\mathrm{N} 15 A$ & $1.218(7)$ & $\mathrm{O} 4 B-\mathrm{N} 15 B$ & $1.224(6)$ \\
$\mathrm{O} 5 A-\mathrm{C} 1 A$ & $1.235(7)$ & $\mathrm{O} 5 B-\mathrm{C} 1 B$ & $1.235(7)$ \\
$\mathrm{O} 6 A-\mathrm{C} 3 A$ & $1.321(7)$ & $\mathrm{O} 6 B-\mathrm{C} 3 B$ & $1.345(7)$ \\
$\mathrm{O} 7 A-\mathrm{C} 3 A$ & $1.198(7)$ & $\mathrm{O} 7 B-\mathrm{C} 3 B$ & $1.201(7)$ \\
$\mathrm{N} 1 A-\mathrm{C} 1 A$ & $1.343(7)$ & $\mathrm{N} 1 B-\mathrm{C} 1 B$ & $1.337(7)$ \\
$\mathrm{N} 1 A-\mathrm{C} 2 A$ & $1.478(6)$ & $\mathrm{N} 1 B-\mathrm{C} 2 B$ & $1.458(6)$ \\
$\mathrm{N} 13 A-\mathrm{C} 13 A$ & $1.468(8)$ & $\mathrm{N} 13 B-\mathrm{C} 13 B$ & $1.474(7)$ \\
$\mathrm{N} 15 A-\mathrm{C} 15 A$ & $1.477(7)$ & $\mathrm{N} 15 B-\mathrm{C} 15 B$ & $1.487(7)$ \\
$\mathrm{C} 1 A-\mathrm{C} 11 A$ & $1.505(8)$ & $\mathrm{C} 1 B-\mathrm{C} 11 B$ & $1.486(8)$ \\
$\mathrm{C} 2 A-\mathrm{C} 4 A$ & $1.523(7)$ & $\mathrm{C} 2 B-\mathrm{C} 3 B$ & $1.509(8)$ \\
$\mathrm{C} 2 A-\mathrm{C} 3 A$ & $1.524(8)$ & $\mathrm{C} 2 B-\mathrm{C} 4 B$ & $1.528(7)$ \\
& & & \\
$\mathrm{C} 1 A-\mathrm{N} 1 A-\mathrm{C} 2 A$ & $123.3(5)$ & $\mathrm{C} 1 B-\mathrm{N} 1 B-\mathrm{C} 2 B$ & $121.9(5)$ \\
$\mathrm{O} 1 A-\mathrm{N} 13 A-\mathrm{O} 2 A$ & $123.9(6)$ & $\mathrm{O} 1 B-\mathrm{N} 13 B-\mathrm{O} 2 B$ & $123.8(6)$ \\
$\mathrm{O} 3 A-\mathrm{N} 15 A-\mathrm{O} 4 A$ & $124.2(7)$ & $\mathrm{O} 3 B-\mathrm{N} 15 B-\mathrm{O} 4 B$ & $125.3(6)$ \\
$\mathrm{O} 5 A-\mathrm{C} 1 A-\mathrm{N} 1 A$ & $122.9(6)$ & $\mathrm{O} 5 B-\mathrm{C} 1 B-\mathrm{N} 1 B$ & $122.5(6)$ \\
$\mathrm{O} 5 A-\mathrm{C} 1 A-\mathrm{C} 11 A$ & $120.0(6)$ & $\mathrm{O} 5 B-\mathrm{C} 1 B-\mathrm{C} 11 B$ & $118.7(6)$ \\
$\mathrm{N} 1 A-\mathrm{C} 1 A-\mathrm{C} 11 A$ & $116.9(6)$ & $\mathrm{N} 1 B-\mathrm{C} 1 B-\mathrm{C} 11 B$ & $118.8(6)$ \\
$\mathrm{N} 1 A-\mathrm{C} 2 A-\mathrm{C} 3 A$ & $108.3(5)$ & $\mathrm{N} 1 B-\mathrm{C} 2 B-\mathrm{C} 3 B$ & $111.6(5)$ \\
$\mathrm{N} 1 A-\mathrm{C} 2 A-\mathrm{C} 4 A$ & $115.9(5)$ & $\mathrm{N} 1 B-\mathrm{C} 2 B-\mathrm{C} 4 B$ & $110.1(5)$ \\
$\mathrm{C} 3 A-\mathrm{C} 2 A-\mathrm{C} 4 A$ & $112.1(5)$ & $\mathrm{C} 3 B-\mathrm{C} 2 B-\mathrm{C} 4 B$ & $113.0(5)$ \\
$\mathrm{O} 6 A-\mathrm{C} 3 A-\mathrm{O} 7 A$ & $123.5(6)$ & $\mathrm{O} 6 B-\mathrm{C} 3 B-\mathrm{O} 7 B$ & $122.7(6)$ \\
$\mathrm{O} 6 A-\mathrm{C} 3 A-\mathrm{C} 2 A$ & $111.2(5)$ & $\mathrm{O} 6 B-\mathrm{C} 3 B-\mathrm{C} 2 B$ & $112.3(5)$ \\
$\mathrm{O} 7 A-\mathrm{C} 3 A-\mathrm{C} 2 A$ & $125.2(6)$ & $\mathrm{O} 7 B-\mathrm{C} 3 B-\mathrm{C} 2 B$ & $124.9(6)$ \\
$\mathrm{C} 2 A-\mathrm{C} 4 A-\mathrm{C} 5 A$ & $115.7(5)$ & $\mathrm{C} 2 B-\mathrm{C} 4 B-\mathrm{C} 5 B$ & $111.5(5)$ \\
$\mathrm{C} 4 A-\mathrm{C} 5 A-\mathrm{C} 6 A$ & $113.3(6)$ & $\mathrm{C} 4 B-\mathrm{C} 5 B-\mathrm{C} 6 B$ & $112.0(6)$ \\
$\mathrm{C} 12 A-\mathrm{C} 11 A-\mathrm{C} 1 A$ & $123.1(6)$ & $\mathrm{C} 12 B-\mathrm{C} 11 B-\mathrm{C} 1 B$ & $125.6(5)$ \\
$\mathrm{C} 16 A-\mathrm{C} 11 A-\mathrm{C} 1 A$ & $116.7(6)$ & $\mathrm{C} 16 B-\mathrm{C} 11 B-\mathrm{C} 1 B$ & $116.6(5)$ \\
& & &
\end{tabular}

$D_{x}=1.471 \mathrm{Mg} \mathrm{m}^{-3}$

Mo $K \alpha$ radiation

Cell parameters from 25 reflections

$\theta=9.3-22.0^{\circ}$

$\mu=0.121 \mathrm{~mm}^{-1}$

$T=294$ (1) K

Plate, colourless

$0.30 \times 0.20 \times 0.05 \mathrm{~mm}$

$h=0 \rightarrow 6$

$k=-12 \rightarrow 12$

$l=-15 \rightarrow 14$

3 standard reflections

intensity decay: $<1 \%$

$(\Delta / \sigma)_{\max }=0.001$

$\Delta \rho_{\max }=0.19 \mathrm{e} \AA^{-3}$

$\Delta \rho_{\min }=-0.18 \mathrm{e} \AA^{-3}$

Extinction correction: SHELXL97

Extinction coefficient: 0.0111 (17)

Absolute structure: Flack (1983)

Flack parameter $=-0.1(16)$

$\begin{array}{lrlr}\mathrm{C} 2 A-\mathrm{N} 1 A-\mathrm{C} 1 A-\mathrm{O} 5 A & -8.9(9) & \mathrm{C} 2 B-\mathrm{N} 1 B-\mathrm{C} 1 B-\mathrm{O} 5 B & 4.3(9) \\ \mathrm{C} 1 A-\mathrm{N} 1 A-\mathrm{C} 2 A-\mathrm{C} 4 A & 70.8(7) & \mathrm{C} 1 B-\mathrm{N} 1 B-\mathrm{C} 2 B-\mathrm{C} 3 B & 81.5(7) \\ \mathrm{N} 1 A-\mathrm{C} 2 A-\mathrm{C} 3 A-\mathrm{O} 7 A & 120.7(6) & \mathrm{N} 1 B-\mathrm{C} 2 B-\mathrm{C} 3 B-\mathrm{O} 7 B & -124.8(7) \\ \mathrm{N} 1 A-\mathrm{C} 2 A-\mathrm{C} 4 A-\mathrm{C} 5 A & 55.1(7) & \mathrm{N} 1 B-\mathrm{C} 2 B-\mathrm{C} 4 B-\mathrm{C} 5 B & 71.3(7)\end{array}$

frequency: $60 \mathrm{~min}$ $P \overline{1}$. The molecule is chiral and space group $P 1$ was chosen and confirmed by the analysis. The absolute configuration is based on ${ }^{1}$ leucine. The crystal diffracted quite weakly but sufficient data (hemisphere) were collected to establish the structure and elucidate the hydrogen bonding interactions. The absolute structure is not reliably determined by this X-ray analysis, but is inferred from the known absolute configuration of the L-leucine used in the synthesis.

Data collection: CAD-4-PC Software (Enraf-Nonius, 1992); cell refinement: SET4 and CELDIM (Enraf-Nonius, 1992); data reduction: DATRD2 in NRCVAX96 (Gabe et al., 1989); program(s) used to solve structure: SHELXS97 (Sheldrick, 1997); program(s) used to refine structure: NRCVAX96 and SHELXL97 (Sheldrick, 1997); software used to prepare material for publication: NRCVAX96, SHELXL97 and PREP8 (Ferguson, 1998).

JFG thanks the Research and Postgraduate Committee of Dublin City University for funding research visits to the University of Guelph (1995-1998) and especially Professor George Ferguson for use of his diffractometer and computer system. PTMK and MOD thank the School of Chemical Sciences for financial support. The purchase of a P4 diffractometer by Dublin City University is appreciated.

\section{References}

Collect, M., Solans, X., Font-Altaba, M. \& Subirana, J. A. (1986). Acta Cryst. C42, 599-601.

Enraf-Nonius (1992). CAD-4-PC Software. Version 1.1. Enraf-Nonius, Delft, The Netherlands.

Ferguson, G. (1998). PREP8. University of Guelph, Canada.

Flack, H. D. (1983). Acta Cryst. A39, 876-881.

Gabe, E. J., Le Page, Y., Charland, J.-P., Lee, F. L. \& White, P. S. (1989). J. Appl. Cryst. 22, 384-387.

Gallagher, J. F., Brady F. \& Murphy, C. (2000). Acta Cryst. C56, 365-368.

Gallagher, J. F., Kenny P. T. M. \& Sheehy, M. J. (1999). Acta Cryst. C55, $1257-$ 1260.

Gallagher, J. F. \& Murphy, C. (1999). Acta Cryst. C55, 2167-2169.

Görbitz, C. H. \& Dalhus, B. (1996). Acta Cryst. C52, 1754-1756.

Harding, M. M. \& Howieson, R. M. (1976). Acta Cryst. B32, 633-634.

Orpen, A. G., Brammer, L., Allen, F. H., Kennard, O., Watson, D. G. \& Taylor, R. (1994). Structure Correlation, Vol. 2, editted by H.-B. Bürgi \& J. D. Dunitz. Weinheim: VCH Publishers.

Sheldrick, G. M. (1997). SHELXS97 and SHELXL97. University of Göttingen, Germany.

Spek, A. L. (1998). PLATON. University of Utrecht, The Netherlands. Vogel, A. I. (1989). Textbook of Practical Organic Chemistry, 5th ed., edited by B. S. Furniss, A. J. Hannaford, P. W. G. Smith \& A. R. Tatchell. Longman Scientific and Technical. 